\title{
DIFFERENTIABLE DYNAMICAL SYSTEMS AND THE PROBLEM OF TURBULENCE
}

\author{
BY DAVID RUELLE
}

1. Conservative and dissipative dynamical systems. The mathematical study of differentiable dynamical systems has its origin in the desire to understand the time evolutions which occur in nature. This relation to physical reality has exercised a strong influence on the progress of the subject. Other strong influences resulted from the internal development of mathematics and physics.

The time evolutions which one observes in nature yield two large classes of dynamical systems. On one hand we have frictionless mechanical systems, or conservative systems. On the other hand we have all kinds of natural systems where some "noble" form of energy is converted to heat; these are the dissipative systems.

Classical mechanics-the study of conservative systems-was initiated by Isaac Newton and has played a central role in the development of Natural Philosophy. In particular it led to the Méthodes nouvelles de la mécanique céleste of Henri Poincaré, and later to the solution of small denominator problems by Kolmogorov, Arnold and Moser. The importance of classical mechanics is due to the existence of nontrivial conservative systems-those of celestal mechanics-which can be investigated with extreme precision both observationally and theoretically.

The dissipative systems have a priori no less interest than the conservative ones. Mathematically they are more general; physically they have widespread occurrence, and display a great variety of phenomena. Unfortunately, dissipative systems do not obey, with great precision, laws as simple as those of celestial mechanics. They are usually continuous systems, requiring an infinite number of parameters for their description. Because of these and other difficulties, a detailed dynamical study of dissipative systems is taking place only now.

2. Viscous flows. Diffusion of heat, chemical reactions, heating of an electrical resistor, are examples of dissipative phenomena. So is the flow of a viscous fluid, where internal friction dissipates mechanical energy into heat.

Viscous flows are among the most remarkable natural phenomena. Whirlpools of the river, waves of the sea, dancing flames of the fire, twisting shapes of the clouds have enchanted poets and fascinated philosophers. To the scientist also viscous flows have something to offer, since understanding

Presented at the Symposium on the Mathematical Heritage of Henry Poincare in April, 1980; received by the editors November 1, 1980.

1980 Mathematics Subject Classification. Primary 76A, 58F. 
hydrodynamic turbulence is a major unsolved problem of theoretical physics. For historical reasons, however, hydrodynamics has fared rather differently from celestial mechanics.

The flow of viscous fluids is described with reasonable precision by the Navier-Stokes equation. This equation has been known for some time, but the mathematical tools and the experimental techniques needed for a detailed study of viscous flows are relatively recent. The mathematical theory of the Navier-Stokes equation begins only in the 1930s with the work of Jean Leray. The experimental study of viscous flow (in particular turbulent flow) is very difficult, and adequate techniques like laser anemometry are only a few years old.

Since the time of Newton it has become progressively more difficult for the same person to be a good mathematician and a good physicist. One man who showed extended competence was G. I. Taylor, who made a remarkable theoretical and experimental study of the flow between rotating concentric cylinders [69]. In general, however, the divorce between physics and mathematics has been unfavorable to the progress of hydrodynamics. At the same time, mathematicians were attracted by the more fundamental aspects of their science, and all the available energies of physicists were drawn by the development of quantum mechanics. Of course, major steps forward have been made by such high class scientists as J. Leray, A. N. Kolmogorov, or E. Hopf, but hydrodynamics has otherwise remained somewhat in the backwaters of the scientific storm of this century.

3. The Navier-Stokes equation. The components of the Navier-Stokes equation are

$$
\frac{\partial v_{i}}{\partial t}+\sum_{j=1}^{d} v_{j} \frac{\partial v_{i}}{\partial x_{j}}=-\frac{\partial p}{\partial x_{i}}+\nu \Delta v_{i}+g_{i}
$$

for $i=1, \ldots, d$. Here $d$ is the number of space dimensions ( $d=3$ usually, but $d=2$ has been much studied). The $v_{i}$ are the components of the velocity field, depending on position $x \in \mathbf{R}^{d}$ and time $t$. The left-hand side of the equation is the acceleration of a particle of fluid. The right-hand side contains the gradient of the pressure $p$, a "dissipative term" with the Laplace operator multiplied by a constant $\nu$ (the viscosity) and an external force $g .{ }^{1}$ We supplement (1) by the incompressibility condition

$$
\sum_{i=1}^{d} \frac{\partial v_{i}}{\partial x_{i}}=0
$$

The fluid is enclosed in a region $\Omega \subset \mathbf{R}^{d}$, and its velocity is imposed on $\partial \Omega$; this is the boundary condition

$$
v_{i}=a_{i} \quad \text { for } i=1, \ldots, d \text { on } \partial \Omega .
$$

If $\Omega$ is bounded, we assume of course that the total flux of $a$ through $\partial \Omega$ vanishes. Usually there is no flow through the boundaries and, since a viscous

\footnotetext{
${ }^{1}$ To be more precise, $p$ and $g$ are the pressure and force divided by the density of the fluid (which will be constant in view of (2)); $\nu$ is the kinematic viscosity.
} 
fluid "sticks" to the wall, (2) will just express that the velocity of the fluid at the boundary is equal to the velocity of the wall (e.g. zero if the wall is fixed). Finally there is an initial condition

$$
v_{i}=v_{i 0} \text { for } t=0 .
$$

The Navier-Stokes equation contains partial derivatives and products which, a priori, make sense only if $v$ is sufficiently smooth. If one multiplies (1) by a smooth function $\phi_{i}(x)$, sums over $i$ and integrates over $x$, one obtains an equation $(1)_{\phi}$. The various terms of $(1)_{\phi}$ are linear or bilinear in $v$, and can be extended by continuity to functional spaces of not necessarily very differentiable functions. If an element of such a functional space verifies all equations $(1)_{\phi}$, in the sense of distributions in $t$, it is called a weak solution of (1). It is advantageous to look first for weak solutions of the Navier-Stokes equations, and later to try to prove their regularity.

For definiteness we assume from now on that $\Omega$ is bounded. To handle the boundary condition (3), one extends $a$ to a divergence-free vector field in $\Omega$, and inserts $v=a+u$ in (1), thus obtaining a new equation for $u$, with $u=0$ on $\partial \Omega$. Let $\mathbf{H}$, resp. $\mathbf{V}$, be the completion of the space of smooth divergencefree vector fields with compact support in $\Omega$, with respect to the $L^{2}$-norm

$$
|u|=\left[\int_{\Omega} d x \sum_{i} u_{i}^{2}\right]^{1 / 2},
$$

resp. the Dirichlet norm

$$
\|u\|=\left[\int_{\Omega} d x \sum_{i j}\left(\frac{\partial u_{i}}{\partial x_{j}}\right)^{2}\right]^{1 / 2} .
$$

The condition $u=0$ on $\partial \Omega$ is expressed by $u \in V$.

One shows that $\mathbf{H}$ is the orthogonal complement of the subspace of gradients in $L^{2}(\Omega)^{3}$. If one takes the projection of (1) orthogonal to gradients, the pressure term disappears and one is left with a functional equation of the form

$$
d u / d t=F_{\mu}(u)
$$

for $u \in \mathbf{V}$. We have left a parameter $\mu$ to describe the intensity of the action exerted on the fluid via the force $g$ and the boundary conditions. A parameter of this sort is usually present in experimental situations (Reynolds number). We have assumed that the action on the fluid is time independent, so that the right-hand side of (5) does not depend on $t$.

We indicate now the main results known on the existence and uniqueness of solutions of the Navier-Stokes equation ${ }^{2}$ for $d=2$ or 3 . We assume that $\partial \Omega$ and $a$ are sufficiently smooth, and that $g$ is square-integrable.

3.1. TheOREM (EXISTENCE). Given $u_{0} \in \mathbf{H}$ there corresponds to it a weak solution $u \in L_{\text {loc }}^{2}((0, \infty), V)$. Furthermore $u$ is weakly continuous $[0, \infty) \mapsto \mathbf{H}$, so that the initial condition $u(0)=u_{0}$ makes sense.

\footnotetext{
${ }^{2}$ For details, see the monographs by Ladyzhenskaya [30], Lions [36], Temam [70], Girault and Raviart [16], and a paper by Foiaş and Temam [13].
} 
3.2. TheOREM (UNIQUENESS). If $d=2$ the weak solution is unique; furthermore it is continuous $[0, \infty) \mapsto \mathbf{H},(0, \infty) \mapsto \mathbf{V}$.

If $d=2$ and $u_{0} \in \mathrm{V}$ the solution is continuous $[0, \infty) \mapsto \mathrm{V}$.

If $d=3$ and $u_{0} \in \mathrm{V}$ there is $t\left(u_{0}\right)>0$ such that the weak solution is unique on $\left[0, t\left(u_{0}\right)\right)$ and continuous $\left[0, t\left(u_{0}\right)\right) \mapsto \mathrm{V}$.

In general, we say that a solution is regular on an interval if it continuous from that interval to $\mathrm{V}$. There can be only one regular solution $u$ on an interval $[0, T]$ with $u(0)=u_{0}$. We write the solution as $u(t)=f^{t} u_{0}$. The maps $\left(f^{t}\right)$, as far as they are defined, satisfy the semigroup property $f^{t+t^{\prime}}=f^{t} \circ f^{t^{\prime}}$. In order to state some analyticity properties of the family $\left(f^{t}\right)$, we introduce the space $\mathbf{D}$ of elements of $\mathbf{V}$ with square-integrable second derivatives. We consider $\mathbf{D}$ as Hilbert space with the norm

$$
\|u\|=\left[\int_{\Omega} d x \sum_{i}\left(\sum_{j} \frac{\partial^{2} u_{i}}{\partial x_{j}^{2}}\right)^{2}\right]^{1 / 2} .
$$

Notice that the inclusion $D \hookrightarrow V$ is compact.

3.3. Theorem (ANAlyticity). ${ }^{3}$ For each $R>0$ there is $T(R)>0$ such that the map $(u, t) \mapsto f^{t} u$ is defined and real-analytic

$$
\{u \in \mathbf{V}:\|u\|<R\} \times(0, T(R)) \mapsto \mathbf{D} .
$$

For $t \in(0, T(R)), f^{t}$ maps $\{u \in \mathrm{V}:\|u\|<R\}$ to a bounded subset of $\mathrm{D}$, and the derivative $D f^{t}$ is an injective linear map $\mathbf{V} \mapsto \mathbf{D}$.

A dynamical system can be obtained by restricting the maps $f^{t}$ to a suitable subset of $\mathbf{V}$. We shall say that an open set $M \subset \mathbf{V}$ is an admissible set (of initial conditions) if every $u \in M$ determines a regular solution on $[0, \infty)$ and there is $T_{0} \geqslant 0$ such that the set $f^{t} M$ is relatively compact in $M$ for $t>T_{0}{ }^{4}$ In particular, if $d=2$, one can show that the ball $\{u \in \mathrm{V}:\|u\|<R\}$ is admissible for sufficiently large $R .^{5}$

3.4. Corollary. Let $M$ be an admissible set of initial conditions. For $t \geqslant T_{0}$, the maps $f^{t}: M \rightarrow M$ are defined, injective, and real-analytic. The semigroup property $f^{t} \circ f^{t^{\prime}}=f^{t+t^{\prime}}$ holds. The maps $(u, t) \mapsto f^{t} u, D f^{t}(u)$ are continuous $M \times\left(T_{0},+\infty\right) \mapsto M, \mathfrak{B}(V)$.

The intersection $\Lambda=\bigcap_{t>T_{0}} f^{t} M$ is compact. If $u \in \Lambda, t>T_{0}$, the operator $D f^{t}(u)$ is compact and injective.

4. The problem of turbulence. While turbulence is often, in practical terms, a well-defined phenomenon, its fundamental nature is still controversial. Call

\footnotetext{
${ }^{3}$ These analyticity properties are explicit or implicit in Fujita and Kato [15], Iooss [25], Foiaş and Temam [13].

${ }^{4}$ These conditions are satisfied if there is $T_{1}>0$ such that every $u \in M$ determines a regular solution on $\left[0, T_{1}\right]$ and $f^{T_{1} M}$ is relatively compact in $M$.

${ }^{5}$ There is $S>0$ such that $\lim \neq \sup _{t \rightarrow \infty}\left\|f^{t} u\right\|<S$ for all $u \in \mathrm{V}$ (see Foiaş and Temam [13, 82]). Take $R>R_{1}>S$ and write $M=\{u \in V:\|u\|<R\}$. If $t_{0}=\frac{1}{2} T(R)$, the set $f^{t_{0}} M$ is bounded in $\mathrm{D}$, hence has compact closure in $\mathrm{V}$. There is thus $t_{1}>0$ such that $f^{t_{1}}\left(f^{t_{0}} M\right) \subset\{u \in$ $V:\|u\|<R_{1}$ \}. Taking $T_{1}=t_{0}+t_{1}$, we see that $f^{T_{1}} M$ is relatively compact in $M$.
} 
$\mu$ the Reynolds number describing the level of "excitation" of a hydrodynamical system (see equation (5)). When $\mu=0$, the system is at rest (asymptotically). When $\mu$ is small, steady states and time periodic motions are observed. When $\mu$ is larger we have the onset of turbulence: the fluid motion becomes nonperiodic in time.

When $\mu$ is sufficiently large, one says that fully developed turbulence is present. The motion is very chaotic and appears to conform to statistical laws of some universality. Such laws have been proposed by Kolmogorov [29]: his "five-thirds law" on the spatial frequency distribution of velocities is well supported by observations. Kolmogorov's theory can be derived from dimensional arguments, and is thus of a somewhat superficial nature. It neglects in particular the phenomenon of intermittency: the fact that much of the velocity gradient $\partial v_{i} / \partial x_{j}$ is concentrated on a small part of physical space. Intermittency has received, at this time, only sketchy theoretical treatment (see Frisch, Sulem and Nelkin [14]). Fully developed turbulence is a three-dimensional phenomenon. For $d=2$, chaotic flow is also observed (by numerical simulation) but the details are rather different.

In his early studies, Leray [34] proposed that turbulence was related to the possible nonuniqueness of weak solutions of the Navier-Stokes equation. A weak solution $t \rightarrow u(t)$ corresponding to a smooth initial condition may at some time $t^{*}$ develop singularities and nonuniqueness. The blowing up of the velocity gradient at singularities may be interpreted as turbulent intermittency. It remains to be seen if nonuniqueness and singularities are really present. Singularities, if they exist, will be on a "small" set ${ }^{6}$ as shown by Scheffer [64], [65] and therefore difficult to detect experimentally.

Leaving the difficult domain of fully developed turbulence, we shall now turn to the onset of turbulence: how does nonperiodicity develop? Here we take the point of view, corroborated by experiment, that the Navier-Stokes equation defines a dynamical system (no breakdown of uniqueness occurs). For $\mu=0$ the dynamical system has an attracting fixed point corresponding to the state of rest. For small $\mu$ the existence of an attracting fixed point corresponding to a steady motion is ensured by the implicit function theorem. When $\mu$ is increased the Hopf bifurcation may give rise to an attracting periodic orbit corresponding to a time-periodic motion. ${ }^{7}$ One may think that further bifurcations will produce attracting tori of increasing dimension $k$, corresponding to quasi-periodic motions with an increasing number $k$ of basic frequencies. When $k$ is large enough, the quasi-periodic motion is interpreted as turbulent. This point of view was proposed by Landau [32] in 1944, and independently by Hopf [23] in 1948. Looking at things more carefully, one finds that the bifurcation from a periodic solution (1-torus) to a 2-torus takes place according to expectations. ${ }^{8}$ But the further bifurcations do not usually take place in a simple manner. If a quasi-periodic motion on a

\footnotetext{
6 The Hausdorff dimension of this set is small.

${ }^{7}$ See Hopf [22], Iooss [24], and for a general discussion Marsden and McCracken [41] which contains an English translation of Hopf's article.

${ }^{8}$ See Naimark [43], Sacker [63], Ruelle and Takens [62], and again Marsden and McCracken for a general discussion.
} 
$k$-torus does occur, it is unstable to perturbations, yielding periodic or (for $k \geqslant 3$ ) nonperiodic motions of all kinds.

In 1971, Takens and myself proposed that nonperiodic motions which are not quasi-periodic describe turbulence. We shall later discuss what is implied. Careful experiments have been made in the past few years. They show that real fluids may be in a steady state, or perform periodic motions, or quasiperiodic motions with commonly two (rarely three) basic frequencies. Furthermore non-quasi-periodic motions are seen as soon as the Reynolds number is large enough. All kinds of bifurcations are observed. The general lesson seems to be that hydrodynamical systems at the onset of turbulence behave very much as generic differentiable dynamical systems in finite dimension. Simple systems of differential equations with arbitrarily chosen coefficients, when studied by digital or analog computer, yield data so analogous to those of hydrodynamical experiments that it is not possible to tell them apart.

5. Sensitive dependence on initial condition and strange attractors. We have stated that a fluid at the onset of turbulence behaves very much like a generic differentiable dynamical system. This assertion is based in part on our mathematical understanding of dynamical systems, in part on the computer study of a number of simple examples. Our mathematical understanding includes the theory of simple bifurcations, and the theory of Smale's Axiom A diffeomorphisms. Computer studies have shown the existence of several new "mathematical phenomena" which would have been very difficult to guess otherwise, and which are in part not understood.

Of particular interest to us is the widespread phenomenon of sensitive dependence on initial condition. This means that a small change in initial condition produces an orbit which deviates exponentially (at least for a while) from the unperturbed orbit. If $\left(f^{t}\right)$ defines a dynamical system on the manifold $M$ (with discrete or continuous time $t$ ), we call attractor a compact set $\Lambda \subset M$ such that $f^{t x} \rightarrow \Lambda$ for $x$ in some neighborhood of $\Lambda$. We assume that $\Lambda$ satisfies some indecomposability condition (like topological transitivity). We shall say that $\Lambda$ is a strange attractor if there is sensitive dependence on initial condition for orbits starting near $\Lambda$. This is not meant as a precise mathematical definition, but rather as a description (subject to revision) of various objects known either mathematically or from computer work. ${ }^{9}$

Reasonably well understood mathematically are the hyperbolic attractors: ${ }^{10}$ the tangent bundle $T_{\Lambda} M$ has a continuous splitting into a contracting subbundle which contracts exponentially under $T f^{t}$ and an expanding subbundle which contracts under $T f^{-t}$ (in the continuous time case there is also a one-dimensional neutral subbundle in the flow direction). If the expanding subbundle is nontrivial, we have sensitive dependence on initial condition, and thus a strange hyperbolic attractor.

\footnotetext{
${ }^{9}$ Notice that Hamiltonian systems may exhibit sensitive dependence on initial conditions, but cannot have strange attractors because of Liouville's theorem.

${ }^{10}$ On hyperbolic attractors (and more generally Axiom A dynamical systems) see Smale [67] and Bowen [2].
} 
A system of differential equations first considered by Lorenz [37] has suggested a mathematical model which is "almost hyperbolic" and fairly well understood. ${ }^{11}$ Nothing has actually been proved, however, about the equations which suggested the model.

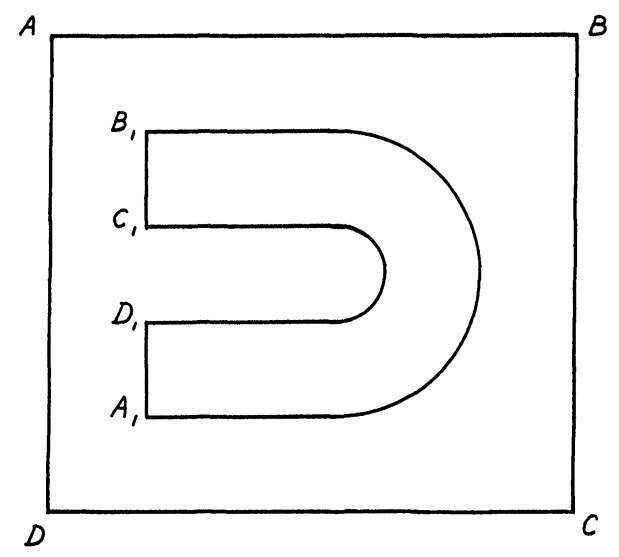

Figure 1.

There are strange hyperbolic attractors for diffeomorphisms of $\mathbf{R}^{2}$, as discovered by Plykin [50], but they are complicated and unobvious. On the other hand very simple folding maps (sending a rectangle $A B C D$ to a horseshoe $A_{1} B_{1} C_{1} D_{1} \subset A B C D$; see Figure $1^{12}$ ) are found to produce sensitive dependence on initial condition. For instance the map $f:(x, y) \rightarrow(y+1-$ $\left.a x^{2}, b x\right)$ has been much studied numerically, and yields for $a=1.4, b=3$ the Hénon attractor. ${ }^{13}$ The computer results suggest that sensitive dependence on initial condition is present for a set of positive Lebesgue measure of values of $(a, b)$, but may be absent for a dense open set (where only periodic orbits would be attracting; see Feit [11]). Mathematically, little is known, but Newhouse ${ }^{14}$ has shown that there is a residual subset of an open (nonempty) set of values of $(a, b)$, for which an infinite number of attracting periodic orbits are present. If a strange attractor $\Lambda$ is thus accompanied by infinitely many periodic attractors, there may be no neighborhood of $\Lambda$ consisting of points $x$ such that $f^{t} x \rightarrow \Lambda$.

We shall not try to improve on our previous definition of a strange attractor to accommodate possible pathologies. It seems preferable to wait for a better mathematical understanding of the Hénon attractor (and other strange attractors known from computer work). What is important is that sensitive dependence on initial condition happens frequently in simple dynamical systems. These systems have a continuous frequency spectrum ${ }^{15}$ by

\footnotetext{
${ }^{11}$ See Guckenheimer [18], Guckenheimer and Williams [19], Williams [72].

12 This is not Smale's horseshoe (which is not an attractor).

13 See Hénon [20], Feit [11], Curry [7].

14 Newhouse [44], [45], and private communication.

15 The frequency spectrum is obtained by frequency analysis of $t \mapsto f^{t} x$. This is much more accessible experimentally than dependence on initial condition.
} 
contrast with the discrete frequency spectrum observed in quasi-periodic systems. The quasi-periodic theory of Landau and Hopf had relied on a torus of large dimension to produce a continuous spectrum by accumulation of many different frequencies. It came somewhat as a surprise to hydrodynamicists that a continuous spectrum would already be produced by a strange attractor in three dimensions (see Monin [42] for an expression of this surprise). Recent experiments by Ahlers [1], Gollub and Swinney [17], and others are in favor of the "strange attractor" theory (first proposed in [62]) rather than that of Landau and Hopf. ${ }^{16}$

The above discussion suggests to call turbulent a hydrodynamical flow with sensitive dependence on initial condition. A quasi-periodic flow would then be nonturbulent (laminar).

6. Ergodic theory of differentiable dynamical systems. The idea of strange attractors has proved physically relevant and fruitful, but their direct geometrical study is discouragingly difficult. Ergodic theory has provided a more rewarding approach, which yields however only "almost everywhere" rather than "everywhere" statements. The first and fundamental result is the multiplicative ergodic theorem of Oseledec. ${ }^{17}$ Consider a differentiable dynamical system $\left(f^{t}\right)$, i.e., a semiflow or semigroup of differentiable maps $M \mapsto M$ where $M$ is a compact manifold. The multiplicative ergodic theorem (or rather a corollary of it) asserts that there is an $\left(f^{t}\right)$-invariant Borel set $\Gamma$, such that $\rho(\Gamma)=1$ for every $\left(f^{t}\right)$-invariant probability measure $\rho$ on $\Gamma$, and

$$
\lim _{t \rightarrow+\infty}\left\|D f^{t}(x) u\right\|=\chi(x, u)
$$

exists for every $x \in \Gamma$. Given $x, \chi(x, u)$ takes only a finite number of values for $u \neq 0$. These values $\lambda_{x}^{(1)}<\cdots<\lambda_{x}^{(s)}$ are called characteristic exponents. More precisely, there is a filtration of $T_{x} M$ by linear subspaces:

$$
0=V_{x}^{(0)} \subset V_{x}^{(i)} \subset \cdots \subset V_{x}^{(s)}=T_{x} M
$$

such that

$$
\lim _{t \rightarrow+\infty}\left\|D f^{t}(x) u\right\|=\lambda_{x}^{(r)} \quad \text { if } u \in V_{x}^{(r)} \backslash V_{x}^{(r-1)} .
$$

We call $m_{x}^{(r)}=\operatorname{dim} V_{x}^{(r)}-\operatorname{dim} V_{x}^{(r-1)}$ the multiplicity of $\lambda_{x}^{(r)}$. With respect to an ergodic measure $\rho$, the characteristic exponents and their multiplicities are almost everywhere constant.

The second fundamental result in the ergodic theory of differentiable dynamical systems is a stable manifold theorem. Taking for simplicity the case of a diffeomorphism, it is asserted that one can choose $\Gamma$ as above and such that, if $x \in \Gamma$ and $\lambda_{x}^{(r)}<\lambda<\min \left(0, \lambda_{x}^{(r+1)}\right)$, then

$$
w_{x}^{\lambda}=\left\{y \in M: \limsup _{t \rightarrow \infty} \frac{1}{t} \log d\left(f^{t} x, f^{t} y\right)<\lambda\right\}
$$

is contained in $\Gamma$, and is the image of $V_{x}^{(r)}$ by an injective immersion tangent

\footnotetext{
${ }^{16}$ See Swinney and Gollub [68] for a review.

17 See Oseledec [46] and Raghunathan [53].
} 
to the identity at $x$, and as smooth as the diffeomorphism $f .{ }^{18}$ In other words, there are manifolds $W_{x}^{\lambda}$ defined for almost all $x$, as smooth as $f$, and tangent at $x$ to $V_{x}^{(r)}$. For $\lambda=0$, we shall call

$$
w_{x}^{-}=\left\{y \in M: \limsup _{t \rightarrow \infty} \frac{1}{t} \log d\left(f^{t} x, f^{t} y\right)<0\right\}
$$

the stable manifold of $x$. The stable manifold for $f^{-1}$ is called unstable manifold.

Stable and unstable manifolds have been much studied in the hyperbolic case; see Hirsch, Pugh, and Shub [21] and references quoted there. Pesin [47]-[49] extended the theory to general diffeomorphisms preserving a smooth measure. Neither the smooth measure nor the invertibility are actually necessary (see Ruelle [58], [59], Ruelle and Shub [61]). However, in the absence of invertibility, one obtains only local stable manifolds (or one has to make some transversality assumption), and the unstable manifold theorem is more complicated to formulate (or one has to assume injectivity).

Let us now leave the technicalities and ask what invariant probability measure. is relevant. Clearly the ergodic average $T^{-1} \Sigma_{t=0}^{T-1} \delta_{f^{\prime} x}$ (or $T^{-1} \int_{0}^{T} d t \delta_{f^{\prime} x}$ ) reproduces every ergodic measure $\rho$ for suitable initial $x$. However, in many cases, the computer evaluation of the above ergodic average gives a single answer (and the probabilistic behavior of a turbulent fluid also of ten seems uniquely defined). The solution of this paradox is that the computer makes roundoff errors (and the time evolution of a fluid is affected by small random perturbations).

In the case of hyperbolic attractors, the occurrence of a single measure in the presence of small stochastic perturbations is well understood. ${ }^{19}$ What happens is that a measure which is stationary under small stochastic perturbations yields, in the limit of zero perturbation, a measure "continuous along the unstable direction". This means that the conditional measures on unstable manifolds are absolutely continuous with respect to the Lebesgue measure on the unstable manifolds. There can be only one $\left(f^{t}\right)$ invariant probability measure $\rho$ with this property on a hyperbolic attractor; it is also characterized by the fact that the measure-theoretic entropy $h(\rho)$ is equal to the sum of the positive characteristic exponents $\Sigma_{\lambda^{(r)}>0} m^{(r)} \lambda^{(r)}$.

I believe that also for nonhyperbolic strange attractors, the stability under small stochastic perturbations drastically reduces the number of relevant ergodic measures. ${ }^{20}$ Counterexamples show that the situation is not as simple as in the hyperbolic case, but some positive results are known. The inequality

$$
h(\rho) \leqslant \int \rho(d x) \sum_{\lambda_{x}^{(r)}>0} m_{x}^{(r)} \lambda_{x}^{(r)}
$$

\footnotetext{
18 The diffeomorphism $f$ must be at least $C^{1+\varepsilon}$; the theorem also holds in the $C^{\infty}$ or $C^{\omega}$ category.

19 The same measure is also obtained as ergodic average for almost all initial $x$ near the attractor with respect to Lebesgue measure. See Sinai [66], Ruelle [54], Bowen and Ruelle [3], Kifer [27], [28].

${ }^{20}$ See Ruelle [56], [57].
} 
holds in general. ${ }^{21}$ If $f$ is a diffeomorphism and $\rho$ is continuous along unstable manifolds, Walters [71] and Katok [26] have shown that

$$
h(\rho)=\int \rho(d x) \sum_{\lambda_{x}^{(r)}>0} m_{x}^{(r)} \lambda_{x}^{(r)}
$$

Furthermore, Pugh and Shub [52] have announced that there is a set of positive Lebesgue measure on the manifold $M$, consisting of points $x$ for which the ergodic average is equal to $\rho$ (one assumes $\rho$ ergodic, with no characteristic exponent equal to zero). It is tempting to believe that such a measure lives on the Hénon attractor. This would for instance explain why computer estimates of the upper characteristic exponent yields reproducibly a single value $\lambda \sim 0.42$. A priori one would expect a scatter of many different values, because the Hénon attractor undoubtedly carries many different ergodic measures with different characteristic exponents.

7. Ergodic theory of the Navier-Stokes equation. We revert to the notation of $\$ 3$, and assume that there exists a nonempty admissible set $M \subset V$ of initial conditions ( $M$ always exists if $d=2$ ). Then $\Lambda=\bigcap_{t>T_{0}} f^{t} M$ is compact. It would be most useful if one could prove (even under restrictive conditions) that $\Lambda$ lies in a finite dimensional $\left(f^{t}\right)$-invariant manifold. ${ }^{22}$ Weaker results have been obtained by Foiaş and Prodi [12], Ladyzhenskaya [31], and Mallet-Paret [38].

7.1. Theorem (MAllet-Paret). ${ }^{23}$ Let $V$ be a separable Hilbert space, and suppose that $\Lambda \subset M \subset \mathbf{V}$ where $\Lambda$ is compact and $M$ open. Let $f: M \rightarrow V$ be $C^{1}$ and satisfy $f \Lambda \supset \Lambda$. Suppose finally that $D f(x)$ is a compact operator for all $x \in \Lambda$. Then $\Lambda$ has finite Hausdorff dimension $H$.

7.2. Corollary (MAÑE). ${ }^{24}$ If $F$ is a linear subspace of $\mathbf{V}$ with dimension $>$ $2 H+1$, then there is a continuous projection $\pi: \mathrm{V} \rightarrow F$ such that its restriction to $\Lambda$ is injective.

In view of Corollary 3.4, Mallet-Paret's theorem applies to the NavierStokes case (where we actually have $f \Lambda=\Lambda$ ). Mañé's corollary shows how to obtain a homeomorphism of $\Lambda$ to a subset of a finite-dimensional Euclidean space.

Using the compactness of the set $\Lambda$ and of the maps $D f(x)$, it is possible to extend the ergodic theory of $\$ 6$ to the present infinite dimensional situation. ${ }^{25}$

\footnotetext{
21 This was first proved by Margulis (quoted by Pesin [47]) for a diffeomorphism with smooth invariant measure. For the case of a general $C^{1}$ map, see Ruelle [55].

22 Apart from the use of the center manifold theorem near a bifurcation, little is known on this problem.

${ }^{23}$ Foias and Temam [13] have derived a direct proof of this result for the Navier-Stokes case, and Mañé [39] for general Banach spaces.

24 See Mañé [39].

${ }^{25}$ I have a Hilbert space extension of the multiplicative ergodic theorem and stable-unstable manifold theorems which weakens the compactness condition on the maps $D f(x)$ and does not assume that they are injective [59]. Independently, Mañe [40] has obtained an extension to compact injective maps in Banach spaces. The application to Navier-Stokes is discussed in Ruelle [60].
} 
7.3. THEOREM. For the Navier-Stokes semiflow, under the assumptions of \$3, the characteristic exponents are defined, and form a sequence tending to $-\infty$ (therefore only a finite number of them can be positive). There are globally defined unstable manifolds; they are finite-dimensional and contained in $\Lambda$. There are locally defined stable manifolds (near $\Lambda$ ); they are finite-codimensional. Stable and unstable manifolds are real-analytic. If some ergodic measure $\rho$ has no characteristic exponent equal to zero the stable and unstable manifolds are $\rho$-almost everywhere transverse to each other.

For a more precise formulation see [59], [40], [60]. We have here assumed that the Navier-Stokes equations defined a smooth dynamical system. It may be, on the contrary, that turbulence is linked to the lack of smoothness of solutions of the Navier-Stokes equations, as proposed by Leray. This does however not seem likely in the weakly turbulent regime. The results quoted above are thus in agreement with the notion that a weakly turbulent viscous fluid behaves like a typical finite-dimensional smooth dynamical system.

8. Further remarks. Many dynamical phenomena have now been observed both in computer simulations and in hydrodynamical experiments. Of special interest is the "Feigenbaum bifurcation" first observed in computer studies of (noninvertible) maps of an interval. Apparently this is a new generic bifurcation which consists in the accumulation of "period doubling" bifurcations. An attracting fixed point can, by a well-understood bifurcation, lose its attracting character and be replaced by an attracting periodic orbit of order 2 . Repeating a similar process $n$ times produces an attracting orbit of order $2^{n}$. What is surprising is that all these bifurcations are often really seen to follow each other, and to converge asymptotically as a geometric sequence. In other words in the space of maps of the interval there seems to exist a "Feigenbaum manifold" of codimension 1, which is the geometric limit of bifurcation manifolds corresponding to period doubling.

M. Feigenbaum, who discovered this phenomenon, has given it a heuristic but highly nontrivial explanation [8], [9] based on the study of an associated dynamical system in a Banach space. A rigorized version of Feigenbaum's arguments is now being worked out (see [6], [33], [4]). It appears that the Feigenbaum bifurcation also occurs in diffeomorphisms and flows (see [5], [10]). In any case, something very much like it is observed in some hydrodynamical experiments (see [35]: this is another example of the occurrence of the same phenomena in hydrodynamical experiments and computer studies, with the same doubts as to their detailed explanation).

To conclude our review it is appropriate to ask what the relation of all this is to the mathematical heritage of Henri Poincaré. His contribution to differentiable dynamical systems is sufficiently impressive, but what about turbulence? I think that it is to the credit of Poincare that he saw that there was a problem, and kept away from it. That he saw a problem is visible from a section entitled "Explication d'un fait expérimental", at the end of his course on the Théorie des tourbillons [51]. He notices that in a liquid flow the vorticity is usually not diffuse, but rather tends to be concentrated in individual whorls. He says that this fact has not been properly explained 
mathematically, and attempts at justifying it by a stability calculation. (He mentions more calculations, which are not reproduced in the book.) The phenomenon considered by Poincaré is two dimensional, and therefore is not really turbulence, but it is clearly related to intermittency. The stability calculations just referred to seem to be as close as Poincaré came to a discussion of turbulence. It is now clear that the knowledge of his time would not allow him to do better. Mathematical physics tries to understand a world of unknown complexity with tools of known limitations. This requires boldness, and modesty. Obviously Henri Poincare lacked neither of these two qualities.

\section{REFERENCES}

1. F. Ahlers, Low-temperature studies of the Rayleigh-Benard instability and turbulence, Phys. Rev. Lett. 33 (1974), 1185-1188.

2. R. Bowen, On Axiom A diffeomorphisms, CBMS Regional Conf. Series, No. 35, Amer. Math. Soc., Providence, R. I., 1978.

3. R. Bowen and D. Ruelle, The ergodic theory of Axiom A flows, Invent. Math. 29 (1975), 181-202.

4. M. Campanino and H. Epstein, On the existence of Feigenbaum's fixed point, Comm. Math. Phys. (to appear).

5. P. Collet, J.-P. Eckmann and H. Koch, Period doubling bifurcations for families of maps on $\mathbf{R}^{n}$ (to appear).

6. P. Collet, J.-P. Eckmann and O. E. Lanford, Universal properties of maps of an interval, Comm. Math. Phys. 76 (1980), 211-254.

7. J. H. Curry, On the Hénon transformation, Comm. Math. Phys. 68 (1979), 129-140.

8. M. J. Feigenbaum, Quantitative universality for a class of nonlinear transformations, J. Statist. Phys. 19 (1978), 25-52.

9. (1979), 669-706.

10. , The transition to aperiodic behavior in turbulent systems, Comm. Math. Phys. (to appear).

11. S. D. Feit, Characteristic exponents and strange attractors, Comm. Math. Phys. 61 (1978), 249-260.

12. C. Foiaş and G. Prodi, Sur le comportement global des solutions nonstationnaires des équations de Navier-Stokes en dimension 2, Rend. Sem. Mat. Univ. Padova 39 (1967), 1-34.

13. C. Foiaş and R. Temam, Some analytic and geometric properties of the solutions of the evolution Navier-Stokes equations, J. Math. Pures Appl. 58 (1979), 339-368.

14. U. Frisch, P.-L. Sulem and M. Nelkin, A simple dynamical model of intermittent fully developed turbulence, J. Fluid Mech. 87 (1978), 719-736.

15. H. Fugita and T. Kato, On the Navier-Stokes initial value problem. I, Arch. Rational Mech. Anal. 16 (1964), 269-315.

16. V. Girault and P.-A. Raviart, Finite element approximation of the Navier-Stokes equations, Lecture Notes in Math., vol. 749, Springer-Verlag, Berlin and New York, 1979.

17. J. P. Gollub and H. L. Swinney, Onset of turbulence in a rotating fluid, Phys. Rev. Lett. 35 (1975), 927-930.

18. J. Guckenheimer, A strange, strange attractor, The Hopf Bifurcation Theorem and Its Applications (J. E. Marsden and M. Mc Cracken, eds.) Springer-Verlag, Berlin and New York, 1976, pp. 368-381.

19. J. Guckenheimer and R. F. Williams, Structural stability of Lorenz attractors, Inst. Hautes Etudes Sci. Publ. Math. 50 (1979), 59-72.

20. M. Hénon, A two-dimensional mapping with a strange attractor, Comm. Math. Phys. 50 (1976), 69-77. 
21. M. Hirsch, C. Pugh and M. Shub, Invariant manifolds, Lecture Notes in Math., vol. 583, Springer-Verlag, Berlin and New York, 1977.

22. E. Hopf, Abzweigung einer periodischen Lösung von einer stationären Lösung eines Differentialsystems, Ber. Math.-Phys. K. Sächs. Akad. Wiss. Leipzig 94 (1942), 1-22.

23. Math. 1 (1948), 303-322.

24. G. Iooss, Existence et stabilité de la solution périodique secondaire intervenant dans les problèmes d'évolution de type Navier-Stokes, Arch. Rational Mech. Anal. 47 (1972), 301-329.

25. Sur la deuxième bifurcation d'une solution stationnaire de systèmes du type

Navier-Stokes, Arch. Rational Mech. Anal. 64 (1977), 339-369.

26. A. Katok, unpublished.

27. Ju. I. Kifer, On the limiting behavior of invariant measures of small random perturbations for some smooth dynamical systems, Dokl. Akad. Nauk SSSR 216 (5) (1974) = Soviet Math. Dokl. 15 (1941), 918-921.

28. __ On small random perturbations of some smooth dynamical systems, Izv. Akad. Nauk SSSR Ser. Mat. 38 (5) (1974), 1091-1115 = Math. USSR-Izv. 8 (1974), 1083-1107.

29. A. N. Kolmogorov, Local structure of turbulence in an incompressible fluid at very high Reynolds numbers, Dokl. Akad. Nauk SSSR 30 (1941), 299-303.

30. O. A. Ladyzhenskaya, The mathematical theory of viscous incompressible flow, 2nd ed., Nauka, Moscow, 1970; 2nd English ed., Gordon and Breach, New York, 1969.

31. _ A dynamical system generated by the Navier-Stokes equations, Zap. Naučn. Sem. Leningrad. Otdel. Mat. Inst. Steklov 27 (1972), 91-115 = J. Soviet Math. 3 (1976), 458-479.

32. L. D. Landau, Turbulence, Dokl. Akad. Nauk SSSR 44 (1944), 339-342.

33. O. E. Lanford, Remarks on the accumulation of period-doubling bifurcations, Mathematical Problems in Theoretical Physics, Lecture Notes in Physics, vol. 116, Springer-Verlag, Berlin and New York, 1980, pp. 340-342.

34. J. Leray, Sur le mouvement d'un liquide visqueux emplissant l'espace, Acta Math. 63 (1934), 193-248.

35. A. Libchaber et J. Maurer, Une expérience de Rayleigh-Bénard de géométrie réduite; multiplication, accrochage et démultiplication de fréquences, J. Physique (to appear).

36. J. L. Lions, Quelques méthodes de résolution des problèmes aux limites non-linéaires, Dunod, Paris, 1969.

37. E. N. Lorenz, Deterministic non-periodic flow, J. Atmosphere Sci. 20 (1963), 130-141.

38. J. Mallet-Paret, Negatively invariant sets of compact maps and an extension of a theorem of Cartwright, J. Differential Equations 22 (1976), 331-348.

39. R. Mañé, On the dimension of the compact invariant sets of certain nonlinear maps, preprint.

40. __ Lyapunov exponents and stable manifolds for compact transformations, preprint.

41. J. E. Marsden and M. McCracken, The Hopf bifurcation and its applications, Applied Math. Sci., vol. 19, Springer-Verlag, Berlin and New York, 1976.

42. A. S. Monin, On the nature of turbulence, Uspehi Fiz. Nauk 125 (1) (1978), 97-122; English transl., Soviet Physiks-Uspekhi 21 (1978), 429-442.

43. Ju. I. Naimark, On some cases of dependence of periodic solutions on parameters, Dokl. Akad. Nauk SSSR 129 (4) (1959), 736-739.

44. S. Newhouse, Diffeomorphisms with infinitely many sinks, Topology 13 (1974), 9-18.

45. _ The abundance of wild hyperbolic sets and non-smooth stable sets for diffeomorphisms, Inst. Hautes Études Sci. Publ. Math. 50 (1979), 102-151.

46. V. I. Oseledec, A multiplicative ergodic theorem. Lyapunov characteristic numbers for dynamical systems, Trudy Moskov. Mat. Obšč. 19 (1968), 197-221.

47. Ya. B. Pesin, Lyapunov characteristic exponents and ergodic properties of smooth dynamical systems with an inveriant measure, Dokl. Akad. Nauk SSSR 226 (4) (1976), 774-777 = Soviet Math. Dokl. 17 (1) (1976), 196-199.

48. Invariant manifold families which correspond to nonvanishing characteristic exponents, Izv. Akad. Nauk SSSR Ser. Mat. 40 (6) (1976), 1332-1379 = Math. USSR-Izv. 10 (6) (1976), 1261-1305.

49. __ Lyapunov characteristic exponents and smooth ergodic theory, Uspehi Mat. Nauk 32 (4) (196) (1977), 55-112 = Russian Math. Surveys 32 (4) (1977), 55-114. 
50. R. V. Plykin, Sources and currents of A-diffeomorphisms of surfaces, Mat. Sb. 94 2(6) (1974), 243-264.

51. H. Poincaré, Théorie des tourbillons, Georges Carré, Paris, 1893.

52. C. C. Pugh and M. Shub, Differentiability and continuity of invariant manifolds, preprint.

53. M. S. Raghunathan, $A$ proof of Oseledec' multiplicative ergodic theorem, Israel J. Math. 32 (1979), 356-362.

54. D. Ruelle, A measure associated with Axiom A attractors, Amer. J. Math. 98 (1976), 619-654.

55. __ An inequality for the entropy of differentiable maps, Bol. Soc. Brasil. Mat. 9 (1978), 83-87.

56. __ Dynamical systems with turbulent behavior, Mathematical Problems in Theoretical Physics, Lecture Notes in Physics, vol. 80, Springer-Verlag, Berlin and New York, 1978, pp. 341-360.

57. , Sensitive dependence on initial condition and turbulent behavior of dynamical systems, Ann. N. Y. Acad. Sci. 316 (1978), 408-416.

58. Ergodic theory of differentiable dynamical systems, Inst. Hautes Études Sci. Publ. Math. 50 (1979), 275-306.

59. __ Characteristic exponents and invariant manifolds in Hilbert space (to appear).

60. __ Measures describing a turbulent flow, Ann. N. Y. Acad. Sci. (to appear).

61. D. Ruelle and M. Shub, Stable manifolds for maps, Lecture Notes in Math., vol. 819, Springer, Berlin, 1980, pp. 389-392.

62. D. Ruelle and F. Takens, On the nature of turbulence, Comm. Math. Phys. 20 (1971), 167-192; note 23 (1971), 343-344.

63. R. J. Sacker, On invariant surfaces and bifurcation of periodic solutions of ordinary differential equations, Thesis, New York University, 1964.

64. V. Scheffer, Turbulence and Hausdorff dimension, Turbulence and Navier-Stokes Equation, Lecture Notes in Math., vol. 565, Springer-Verlag, Berlin and New York, 1979, pp. 94-112. 65. , The Navier-Stokes equations on a bounded domain, Comm. Math. Phys. 73 (1980), 1-42.

66. Ia. G. Sinai, Gibbs measure in ergodic theory, Uspehi Mat. Nauk 27 (4) (1972), 21-64 = Russian Math. Surveys 27 (4) (1972), 21-69.

67. S. Smale, Differentiable dynamical systems, Bull. Amer. Math. Soc. 73 (1967), 747-817.

68. H. L. Swinney and J. P. Gollub, The transition to turbulence, Physics Today 31 (1978), 41-49.

69. G. I. Taylor, Stability of a viscous liquid contained between two rotating cylinders, Philos. Trans. Roy. Soc. London Ser. A 223 (1923), 289-343.

70. R. Temam, Navier-Stokes equations, rev. ed., North-Holland, Amsterdam, 1979.

71. P. Walters, Characteristic exponents and the entropy of diffeomorphisms, preprint.

72. R. F. Williams, The structure of Lorenz attractors, Inst. Hautes Études Sci. Publ. Math. 50 (1979), 73-99.

Institut des Hautes Études Scientifiques, BuRes-SUR-YvetTe, France 